\title{
ANELAMENTO E PACLOBUTRAZOL NA PRODUÇÃo E ABSORÇÃO DE NUTRIENTES EM PEREIRA (Pyrus communis L.) CULTIVAR PACKHAM'S TRIUMPH ${ }^{1}$
}

\author{
TAKESHI IUCHI ${ }^{2}$,VERALÚCIAIUCHI ${ }^{3}$, FLÁVIO GILBERTO HERTER $^{4}$, EMIILIO BRIGHENTI $^{5}$
}

RESUMO - Foram conduzidos dois experimentos para estudar o efeito do anelamento e do paclobutrazol na produção e absorção de macronutrientes em pereira cv Packham’s Triumph, no munícipio de São Joaquim-SC, localizado a 1.400 m de altitude, em pomar comercial, utilizando plantas adultas.O delineamento utilizado no primeiro e segundo foi o de blocos casualizados. No primeiro estudo, foram utilizados sete tratamentos: 1)testemunha; 2) 1,5 g /planta do paclobutrazol, aplicados no solo; 3) 3,0 g/planta de paclobutrazol, aplicados no solo; 4 ) 4,5 g/planta de paclobutrazol, aplicados no solo; 5) anelamento simples (1 anel); 6) anelamento duplo realizado (dois anéis), e 7 ) anelamento pleno (um anelamento seguido de outro anelamento no mesmo local após a cicatrização do primeiro). No segundo experimento, os tratamentos foram: 1) - testemunha; 2) $2 \mathrm{~g} /$ planta de paclobutrazol, aplicados o solo; 3 ) 4 g/planta de paclobutrazol, aplicados no solo; 4 ) - 1.000 ppm de paclobutrazol em aplicação foliar; 5 ) 2.000 ppm de paclobutrazol em aplicação foliar; 6 ) anelamento simples, e 7) anelamento duplo. A maior produção foi observada no anelamento pleno e no anelamento duplo. $\mathrm{O}$ anelamento teve maior efeito na produção do que o paclobutrazol, e este via solo teve maior efeito do que a aplicação foliar. Os tratamentos não influenciaram nos níveis de nutrientes no fruto, com exceção do potássio. O maior teor de potássio foi encontrado na testemunha que apresentou o maior tamanho de frutos.

Termos para indexação: Pyrus communis L., anelamento, reguladores de crescimento.

\section{THE RING AND PACLOBUTRAZOL IN MINERAL NUTRITION ON PEAR (Pyrus communis L.) PACKHAM'S TRIUMPH CULTIVAR}

\begin{abstract}
Two trials was conducted to study the effect of the ring and paclobutrazol in the production and mineral nutrition in pear tree Packham's Triumph cv, in São Joaquim city, SC, south of Brazil, located in 1,400 m of altitude, in a commercial orchard, using adult plants.The experimental delineation used in the trials was of randomized blocks. In the first trial it was studied 7 treatments : 1)

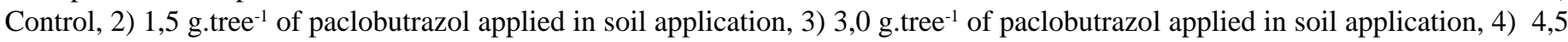
g.tree ${ }^{-1}$ of paclobutrazol applied in soil application, 5) ring (one ring), 6) double ring and 7) full ring (one ring followed by another ring in the same place after cicatrization ).In the second trial, it was studied: 1) control, 2) 2,0 g.tree-1 of paclobutrazol applied in soil application 3) 3,0 g.tree $\mathrm{e}^{-1}$ of paclobutrazol applied in soil application, 4) $1000 \mathrm{mg} . \mathrm{l}^{-1}$ paclobutrazol by foliar application, 5) $2000 \mathrm{mg} .1$ ${ }^{-1}$ of paclobutrazol by foliar application, 6) one ring and 7) double ring .The highest production was observed in the full and double ring. The ring had greater effect in the production and the paclobutrazol soil application had greater effect than the foliar application. The treatments did not influence the nutrients levels in the fruit with exception of the potassium. The highest potassium level was found in the control which showed the biggest fruits.
\end{abstract}

Index terms: Pyrus communis L., ring , plant growth regulators.

\section{INTRODUÇÃO}

A produção brasileira de pêra, na média do período de 2001 a 2005, foi de 20.230 toneladas, destacando-se o Rio Grande do Sul como o principal produtor, com $45,7 \%$ do total. O restante dividiu-se entre os Estados de São Paulo (22,8\%) Paraná (10,9\%), Santa Catarina (10,0\%), Minas Gerais (4\%) e Rio de Janeiro $(1,2 \%)$. A pêra fresca é a fruta importada em maior quantidade pelo Brasil. De 2001 a 2005, foram importadas, em média, 90.296,7 toneladas e gastos U\$ 41.235,4. Esses valores significaram, respectivamente, $38,38 \%$ da quantidade total de frutas importadas e 26,16\% do valor pago. As pequenas áreas de plantio estão localizadas em São Paulo, Minas Gerais, Paraná, Rio Grande do Sul e Santa Catarina (Fioravanço, 2007).

Vários autores afirmam que a principal causa que impede a produção da pereira no Brasil, é a falta de cultivares adaptadas, mas observa-se que a falta de manejo adequado, como a poda e arqueamento de ramos são fatores da baixa produtividade da pereira. Na região serrana de Santa Catarina, existe um grande potencial para o plantio de cultivares mais exigentes em horas de frio hibernal, principalmente as pereiras do tipo européias. Há grande interesse pelo plantio de pereira européia ou japonesa, principalmente pelos pequenos produtores, fazendo-se

${ }^{1}$ (Trabalho 279-07). Recebido em : 23-11-2007. Aceito para publicação em: 31-10-2008.

${ }^{2}$ Eng. Agr., Doutor Embrapa Clima Temperado, Estação Experimental de Fruticultura Temperado CNPUV Vacaria-RS Caixa Postal 1513. CEP 952000000.iuchi@cpact.embrapa.br

${ }^{3}$ Eng. Agr., Doutor EPAGRI, Estação Experimental de São Joaquim, caixa postal 81 São Joaquim-SC. CEP- 88600-000.iuchi@yahoo.com.br ${ }^{4}$ Eng. Agr. Doutor, Embrapa Clima Temperado, Pelotas-RS, caixa postal 403, CEP 96001-970.herter@cpact.embrapa.br.

${ }^{5}$ Eng. Agr., Mestre EPAGRI, Estação Experimental de São Joaquim, caixa postal 81 São Joaquim-SC. CEP- 88.600- 000.brighrent@epagri.com.br

Rev. Bras. Frutic., Jaboticabal - SP, v. 30, n. 4, p. 857-861, Dezembro 2008 
necessário, então, geração de tecnologias para a cultura.

Os principais problemas de frutificação estão relacionados com período juvenil prolongado, abortamento de gemas florais, pouca formação de gemas florais e baixa frutificação devido à forte competição de fotoassimilados por pontos de crescimento (Leopold \& Kridemann, 1975). Fisiologicamente, o estado juvenil pode ser descrito como o período em que as plantas têm aumento exponencial em tamanho, quando o processo de floração e frutificação não pode ser induzido, e a morfologia das folhas e gemas são características.

Para diminuir o período juvenil e, conseqüentemente, iniciar a produção, vários métodos podem ser utilizados. A poda é a prática cultural mais comum para reduzir o crescimento vegetativo e manter a capacidade produtiva. Uma comparação de custo de poda versus reguladores de crescimento, para aumentar a eficiência de produção, mostrou que a poda resultou em uma perda na renda líquida devido à redução na superfície de produção, e além do custo, os reguladores de crescimento aumentaram o retorno econômico pela diminuição do custo de produção (Elfving, 1986).

O paclobutrazol, um retardante de crescimento contendo (2rs,3rs) 1-(4-clorofenil) 4,4-dimetil2 - (1h1,2,4-triazol il) pentan 3 ol, foi primeiramente utilizado via foliar em dosagem de 500 a 8.000 g/l (William's \& Edgerton, 1983; Tukey, 1981). Entretanto, trabalhos posteriores demonstraram que esse produto tinha melhor absorção pelo sistema radicular. Além do paclobutrazol, outros redutores de crescimento foram estudados, como clormequat Cycocel (Maas, 2005), prohexadione cálcio (Iuchi \& Iuchi, 2006 a,b; Maas, 2005; Deckers et al., 2005).

Métodos físicos e químicos também são utilizados para o controle do crescimento. Dentre estes, o entalhamento, o anelamento e o estrangulamento agem pela interrupção da translocação no floema e causam armazenamento dos carboidratos na porção do ramo acima do corte, assim como hormônios de crescimento produzidos pelo meristema apical e folhas jovens, promovendo a indução floral ou a frutificação ( Marafon et al., 2008; Yamanishi et al., 1995).

Com a finalidade de aumentar a produção de frutos e estudar a absorção de nutrientes, conduziuram-se estes experimentos com a utilização de anelamento e do paclobutrazol.

\section{MATERIAL E MÉTODOS}

Foram conduzidos dois experimentos no município de São Joaquim-SC, com a pereira cultivar Packham's Triumph sobre o porta-enxerto Pyrus caleryana. O pomar comercial está localizado a $1.400 \mathrm{~m}$ de altitude, nas coordenadas geográficas com latitude $28^{\circ} 18^{\prime} \mathrm{S}$ e longitude $49^{\circ} 56^{\prime} \mathrm{W}$.

$\mathrm{O}$ delineamento experimental utilizado no primeiro experimento foi em blocos casualizados, com 7 tratamentos e 6 repetições, sendo cada parcela constituída por 1 planta. Os tratamentos foram: 1) testemunha; 2) 1,5 g /planta do paclobutrazol, via solo, aplicados em 28-09-2000 e 26-11-2001; 3) $3,0 \mathrm{~g} /$ planta de paclobutrazol, aplicados no solo em 28-09-2000 e 26-11-2001; 4) 1.000 ppm de paclobutrazol, em aplicação foliar de 4,5 g/planta de paclobutrazol, aplicados no solo em 28-09-2000 e 26-11-2001; 5) anelamento simples realizado em 28-09-2000 e 21-11-2001; 6) - anelamento duplo, realizados em 28-09-2000 e 21 11-2001, e 7) anelamento pleno, realizado, em 28-09-2000, 22-112000 e 21-11-2001, consistindo de dois anelamentos realizados no primeiro ano, sendo após a cicatrização realizado novo anelamento. As plantas foram plantadas no inverno do ano de 1992.

O delineamento experimental utilizado no segundo experimento também foi em blocos casualizados, com 7 tratamentos, com 6 repetições, e cada parcela constituída de 1 planta. Constituiu-se dos seguintes tratamentos: 1) testemunha (0 g de paclobutrazol); 2) $2 \mathrm{~g} /$ planta de paclobutrazol, aplicados no solo em 01-12-2000 e 21-11-2001;3) 4 g/planta de paclobutrazol aplicados no solo em 01-12-2000 e 21-11-2001; 4) 1.000 ppm de paclobutrazol em aplicação foliar em 01-12-2000 e 21-11-2001; 5) 2.000 ppm de paclobutrazol em aplicação foliar em 01-12-2000 e 21-11-2001; 6) anelamento simples realizado em 01-12-2000 e 2111-2001, e 7) anelamentos duplos realizados em 01-12-2000 e 2111-2001. Os anelamentos foram realizados com anelador, a $20 \mathrm{~cm}$ acima do solo, retirando-se uma largura de $8 \mathrm{~mm}$ de casca em toda a circunferência do tronco. Neste experimento, as plantas utilizadas foram plantadas em 1995.

As colheitas foram realizados em 14-02-2002 e 15-02-2002. Foram coletados 10 frutos/parcela para realizar a análise de macronutrientes (nitrogênio, fósforo, cálcio e magnésio). O comprimento dos ramos foi medido em 03-07-2002.

Os dados foram submetidos à análise de variância e à comparação de média, pelo teste de Duncan, ao nível de 5\% de probabilidade.

\section{RESULTADOS E DISCUSSÃO}

A análise da variância revelou efeito significativo ao nível de $1 \%$ de probabilidade para o número de frutos, no primeiro experimento. Houve aumento de 33,7 frutos.planta ${ }^{-1}$ na testemunha para 101,7 frutos planta ${ }^{-1}$ no anelamento pleno, que não diferiu do anelamento duplo (92,0 planta $\left.^{-1}\right)$ (Tabela 1). $\mathrm{O}$ paclobutrazol e o anelamento simples não tiveram efeito significativo sobre o número de frutos. Porém destaca-se que o melhor efeito foi na dosagem de $3,0 \mathrm{~g} \mathrm{planta}^{-1}$, revelando que as aplicações mais cedo não aumentaram o número de frutos. No segundo experimento, a análise da variância revelou efeito significativo do número de frutos ao nível de $1 \%$ de probabilidade (Tabela 2). Houve aumentos de 73,8 frutos planta $^{-1}$ na testemunha para 183,7 frutos planta ${ }^{-1}$ no anelamento duplo.

O aumento da produção de frutos deve-se, provavelmente, ao acúmulo de fotoassimilados, mudança na partição de assimilados, alocando mais na parte reprodutiva, formação de gemas florais, fato constatado por MARAFON et al. (2008), e menor crescimento da parte vegetativa. $O$ anelamento provoca bloqueio no floema, os assimilados não se translocam abaixo deste. Há mudança na translocação de reguladores de crescimento, provocando mudanças físiológicas na planta (Leopold \& Kridemann, 1975).

O peso dos frutos foi afetado pelos tratamentos, ao nível 
de $5 \%$ de probabilidade, no primeiro experimento (Tabela 1 ), sendo que a maior produção de frutos foi obtida pelo anelamento pleno, com a produção de $17,1 \mathrm{~kg}$.planta ${ }^{-1}$, enquanto a testemunha produziu $7,2 \mathrm{~kg}$ planta $^{-1}$. No segundo experimento, a produção de frutos aumentou de $17,5 \mathrm{~kg}$. planta $^{-1}$ na testemunha para 34,9 kg. planta ${ }^{-1}$, no anelamento duplo (Tabela 2).

A variação de produção em função do anelamento devese à intensidade em que foi realizado o corte. $\mathrm{O}$ tempo em que o corte permanece bloqueando o transporte de fotoassimilados é fator que determina a intensidade do efeito do anelamento, e quanto maior o tempo deste, maior será o efeito. No primeiro experimento, quando ocorreu a cicatrização do anelamento pleno, realizou-se nova abertura da casca, aumentando a redução do crescimento e o acúmulo de reservas (Brickel, 1979).

A aplicação de paclobutrazol via solo, no segundo experimento, foi mais tardia do que no primeiro; em razão disto, houve maior efeito. A resposta do paclobutrazol à aplicação via foliar foi pequena (Tabela 2). Esse resultado está de acordo com Curry \& William's (1986) e William's \& Edgerton (1983), que relataram que o paclobutrazol tem melhor efeito com aplicação via solo. Os efeitos com outros reguladores de crescimento na redução do crescimento e no aumento da frutificação foram observados por Glenn \& Miller (2005), Iuchi Iuchi (2006 a) e Iuchi \& Iuchi (2006 b).

$\mathrm{O}$ aumento no número e no peso de frutos por planta deve-se à mudança na partição de assimilados onde o anelamento alocou maior quantidade de fotoassimilados na formação e crescimento de frutos. O anelamento tornou o fruto um dreno mais forte e competitivo. A análise da variância revelou efeito significativo do tratamento a nível de $5 \%$ de probabilidade, a nível de peso médio de frutos no primeiro experimento, sendo que o maior peso médio foi observado quando se adotou o anelamento simples. Isto está relacionado com o número de frutos na planta (Tabela 2). A redução de peso médio dos frutos com o aumento do número de frutos concorda com relatos de alguns autores, que constataram que o aumento da frutificação pode diminuir o peso médio do fruto (Curry \& William’s, 1986). Já no segundo experimento, o número de frutos e o peso total de frutos não afetaram o peso médio, o que pode ser devido às reservas terem sido suficientes para garantir o aumento do número de frutos.

A firmeza e o teor de sólidos solúveis totais no primeiro e segundo experimentos não foram afetados pelos tratamentos (Tabelas 1 e 2). Esse resultado difere do observado por Curry \& William's (1986), provavelmente porque a carga de frutos não atingiu níveis que afetassem o armazenamento de fotoassimilados.

$\mathrm{O}$ anelamento diminuiu o crescimento de ramos de pereira (Tabelas 1 e 2).Esse resultado concorda com os obtidos por MEDJOUB et al. (2005), e os ramos mais curtos permitem maior penetração de luz nas partes inferior e interior do dossel, e aumentam a produção de gemas florais (Marafon et al., 2008) e, em conseqüência, aumentam a produtividade. A redução no crescimento de ramos foi obtida com a aplicação de prohexadione cálcio (Glenn \& Miller, 2005; Prive et al., 2004; Warnier, 2003). Outro efeito do anelamento pode ser a quebra de dominância apical de ramos, melhorando a formação de gemas florais.
No experimento 1 , os tratamentos de anelamento e o paclobutrazol não afetaram significativamente os teores dos nutrientes, nitrogênio, fósforo, potássio, cálcio e magnésio, e as relações N/Ca, $\mathrm{K} / \mathrm{Ca}$ e $\mathrm{K}+\mathrm{Mg} / \mathrm{Ca}$ (Tabela 3). O teor de nitrogênio nos frutos foi de aproximadamente $486 \mathrm{mg} / \mathrm{kg}$ de polpa fresca, com teores similares ao obtido por Luz et al. (2005), na pêra cv.Rocha, e teores similares aos de maçã das cultivares Gala e Golden Delicious, e superior ao da cultivar Fuji (Suzuki \& Argenta, 1994). Esses teores são ligeiramente superiores aos normais citados por Nava et al. (2002) e ao apresentado por Nachtgall et al. (2004). Os teores de fósforo obtidos no fruto no experimento foram similares aos obtido na pêra cv. Rocha por Luz et al. (2005). Se comparada com a maçã, esses teores foram normais (maior que $100 \mathrm{mg} / \mathrm{kg}$ ) (Nachtgall et al., 2004; Nava et al., 2002; Suzuki \& Argenta, 1994). Os teores de potássio foram afetados significativamente pelos tratamentos, ao nível de $5 \%$ de probabilidade (Tabela 4), sendo que o teor encontrado nos frutos foi similar aos obtidos por Luz et al. (2005) .Esses teores foram ainda semelhantes aos encontrados por Nachtgall et al.(2004), Nava et al. (2002) e Suzuki \& Argenta (1994). Em maçã o teor de cálcio no fruto está abaixo da concentração encontrada na pêra, por Luz et al. (2005) (Tabela 4). O valor obtido foi considerado normal, segundo Nava et al. (2002), que consideram teores normais acima de $40 \mathrm{mg} / \mathrm{kg}$. Na colheita e após o período de armazenamento, não se observaram sintomas de distúrbio fisiológico nos frutos. Em razão disso, acredita-se que os teores são normais. $O$ teor de magnésio foi similar ao encontrado na pêra cv Rocha por Luz et al. (2005), e similar ao obtido em maçã por Nava et al. (2002). Esses resultados são superiores ao de Suzuki \& Argenta (1994), que observaram teor de $50 \mathrm{mg} \cdot \mathrm{kg}^{-1}$. Quanto à relação potássio/cálcio, atingiu níveis abaixo do normal, de acordo com Nava et al. (2002). No entanto, não apresentaram sintomas de distúrbios fisiológicos no fruto. Os teores de nitrogênio, fósforo, cálcio e magnésio, e as relações N/Ca, K/Ca e $\mathrm{K}+\mathrm{Mg}$ /cálcio não apresentaram diferenças significativas entre os tratamentos no segundo experimento (Tabela 4). O nível de nitrogênio foi similar aos obtidos por Luz et al. (2005). Os níveis de fósforo obtidos foram similares aos obtidos por Luz et al. (2005), Nava et al. (2002) e Suzuki \& Argenta (1994) na maçã Gala, porém superior à cultivar Golden Delicious e Fuji. A relação K/Ca obtido no presente experimento foi de $26,7 \mathrm{mg} \cdot \mathrm{kg}^{-1}$, um pouco abaixo do normal, que é o padrão superior a $30,0 \mathrm{mg} \cdot \mathrm{kg}^{-1}$ (Nava et al., 2002). 
TABELA 1 - Produção, comprimento de ramos, peso médio, firmeza e sólidos solúveis totais (SST) dos frutos da pereira cv Packham’s Triumph sob efeito do anelamento simples, duplo e pleno, e doses de paclobutrazol, durante o ciclo de 2001/2002.

\begin{tabular}{|c|c|c|c|c|c|c|}
\hline \multirow[t]{2}{*}{ Tratamento } & \multicolumn{2}{|c|}{ Produção de frutos } & \multirow{2}{*}{$\begin{array}{c}\text { Peso } \\
\text { médio } \\
(\mathrm{g})\end{array}$} & \multirow{2}{*}{$\begin{array}{c}\text { firmeza } \\
\left(\mathrm{Lb} / \mathrm{pol}^{2}\right)\end{array}$} & \multirow{2}{*}{$\begin{array}{c}\text { SST } \\
\left({ }^{\circ} \text { brix }\right)\end{array}$} & \multirow{2}{*}{$\begin{array}{l}\text { Comp. } \\
(\mathrm{cm})\end{array}$} \\
\hline & número & (Kg/planta) & & & & \\
\hline Teste munha & $33,7 \mathrm{~b}$ & $7,18 \mathrm{c}$ & $212,2 \mathrm{ab}$ & 15,6 & 13,1 & $19,11 b$ \\
\hline Paclobutrazol 1,5 g/planta & $43,8 \mathrm{~b}$ & $8,27 \mathrm{bc}$ & $187,2 \mathrm{~b}$ & 16,0 & 12,8 & $27,51 \mathrm{c}$ \\
\hline Paclobutrazol 3,0 g/planta & $56,7 \mathrm{ab}$ & $11,46 a b c$ & $210,5 \mathrm{ab}$ & 16,6 & 12,8 & $22,60 \mathrm{c}$ \\
\hline Paclobutrazol 4,5 g/planta & $38,2 \mathrm{~b}$ & $7,88 \mathrm{c}$ & $208,6 \mathrm{ab}$ & 16,4 & 12,1 & $28,59 \mathrm{c}$ \\
\hline Anelamento simples & $44,5 \mathrm{~b}$ & $10,22 \mathrm{abc}$ & $235,3 \mathrm{a}$ & 16,4 & 12,4 & $8,11 \mathrm{a}$ \\
\hline Anelamento duplo & $92,0 \mathrm{a}$ & $15,99 \mathrm{ab}$ & $177,0 \mathrm{~b}$ & 16,1 & 12,1 & $5,88 \mathrm{a}$ \\
\hline Anelamento pleno & $101,7 \mathrm{a}$ & $17,12 \mathrm{a}$ & $171,6 \mathrm{~b}$ & 16,3 & 12,2 & $7,93 \mathrm{a}$ \\
\hline $\mathrm{F}$ & $1 \%$ & $5 \%$ & $5 \%$ & NS & NS & $1 \%$ \\
\hline
\end{tabular}

As médias seguidas da mesma letra na mesma coluna não diferem entre si, pelo teste de Duncan, ao nível de 5\% de probabilidade. NS = não-significativo.

TABELA 2 - Produção, comprimento de ramos, peso médio, firmeza e sólidos solúveis totais (SST) dos frutos da pereira cv Packhaḿs Triumph sob efeito do anelamento simples, duplo e pleno, e doses de paclobutrazol aplicados via solo e foliar, durante o ciclo de 2001/2002.

\begin{tabular}{|c|c|c|c|c|c|c|}
\hline \multirow[t]{2}{*}{ Tratamento } & \multicolumn{2}{|c|}{ Produção de frutos } & \multirow{2}{*}{$\begin{array}{l}\text { Peso médio } \\
\text { (g) }\end{array}$} & \multirow{2}{*}{$\begin{array}{l}\text { firmeza } \\
\mathrm{Lb} / \mathrm{pol}^{2}\end{array}$} & \multirow{2}{*}{$\begin{array}{l}\text { SST } \\
\text { (brix ) }\end{array}$} & \multirow{2}{*}{$\begin{array}{l}\text { Comp. } \\
(\mathrm{cm})\end{array}$} \\
\hline & número & (kg/planta) & & & & \\
\hline Testemunha & $73,8 \mathrm{c}$ & $17,6 \mathrm{c}$ & 233,7 & 12,3 & 16,2 & $20,2 \mathrm{~b}$ \\
\hline Paclobutrazol 2g/planta solo & $68,7 \mathrm{c}$ & $15,3 \mathrm{c}$ & 230,3 & 12,8 & 16,1 & $18,4 \mathrm{~b}$ \\
\hline Paclobutrazol 4 g/planta solo & $129,0 \quad a b$ & $29,8 \mathrm{ab}$ & 242,9 & 12,3 & 16,5 & $22,4 \mathrm{~b}$ \\
\hline Paclobutrazol $1000 \mathrm{mg} / \mathrm{l}$ foliar & $97,7 \mathrm{bc}$ & $19,6 \mathrm{bc}$ & 201,1 & 13,0 & 15,8 & $20,4 \mathrm{~b}$ \\
\hline Paclobutrazol $2000 \mathrm{mg} / \mathrm{l}$ foliar & $94,3 \mathrm{ab}$ & $20,8 \mathrm{bc}$ & 220,1 & 12,0 & 16,3 & $25,3 \mathrm{~b}$ \\
\hline Anelamento simples & $149,0 \mathrm{ab}$ & $32,0 \mathrm{ab}$ & 227,2 & 12,4 & 15,2 & $10,1 \mathrm{a}$ \\
\hline Anelamento duplo & $183,7 \mathrm{a}$ & 34,9 a & 191,1 & 11,8 & 15,6 & $7,9 \mathrm{a}$ \\
\hline $\mathrm{F}$ & $1 \%$ & $1 \%$ & NS & NS & NS & $1 \%$ \\
\hline
\end{tabular}

As letras seguidas da mesma letra na mesma coluna não diferem entre si, pelo teste de Duncan, ao nível de 5\% de probabilidade. NS $=$ não-significativo.

TABELA 3 - Teor de macronutrientes na polpa fresca da pêra (mg.kg -1) cv Packham’s Triumph sob efeito do anelamento simples,duplo e pleno, e doses de paclobutrazol, durante o ciclo de 2001/2002.

\begin{tabular}{lcccccccc}
\hline Tratamento & $\mathrm{N}$ & $\mathrm{P}$ & $\mathrm{K}$ & $\mathrm{Ca}$ & $\mathrm{Mg}$ & $\mathrm{N} / \mathrm{Ca}$ & $\mathrm{K} / \mathrm{Ca}$ & $\mathrm{K}+\mathrm{Mg} / \mathrm{Ca}$ \\
& & & & & & & & \\
\hline Testemunha & 413,8 & 132,8 & 1124,7 & 53,7 & 64,3 & 8,7 & 22,8 & 24,2 \\
Paclobutrazol 1,5 g/planta & 508,0 & 139,5 & 1244,7 & 52,0 & 73,2 & 9,9 & 25,1 & 28,9 \\
Paclobutrazol 3,0 g/planta & 504,7 & 129,8 & 1214,3 & 61,3 & 80,3 & 8,6 & 20,7 & 22,1 \\
Paclobutrazol 4,5 g/planta & 505,8 & 137,7 & 1219,0 & 54,0 & 73,8 & 9,5 & 22,8 & 24,2 \\
Anelamento simples & 483,1 & 134,0 & 1292,8 & 63,5 & 73,0 & 7,9 & 20,8 & 21,4 \\
Anelamento duplo & 540,8 & 127,8 & 1124,8 & 69,7 & 77,0 & 8,0 & 16,7 & 17,9 \\
Anelamento pleno & 447,0 & 133,8 & 1145,2 & 54,2 & 75,0 & 9,2 & 22,7 & 24,2 \\
\multicolumn{1}{c}{$\mathrm{F}$} & $\mathrm{NS}$ & $\mathrm{NS}$ & $\mathrm{NS}$ & $\mathrm{NS}$ & $\mathrm{NS}$ & $\mathrm{NS}$ & $\mathrm{NS}$ & $\mathrm{NS}$ \\
\hline
\end{tabular}

NS = não-significativo

TABELA 4- Teor de macronutrientes na polpa fresca da pêra $\left(\mathrm{mg}_{\mathrm{kg}} \mathrm{kg}^{-1}\right) \mathrm{cv}$ Packham's Triumph sob efeito anelamento simples, duplo e pleno, e doses de paclobutrazol aplicados via solo e foliar, durante o ciclo de 2001/2002.

\begin{tabular}{lcccccccc}
\hline Tratamento & $\mathrm{N}$ & $\mathrm{P}$ & $\mathrm{K}$ & $\mathrm{Ca}$ & $\mathrm{Mg}$ & $\mathrm{N} / \mathrm{Ca}$ & $\mathrm{K} / \mathrm{Ca}$ & $\begin{array}{c}\mathrm{K}+\mathrm{Mg} / \\
\mathrm{Ca}\end{array}$ \\
& & & & & & & & 22,5 \\
Testemunha & 455,0 & 114,7 & $1280,3 \mathrm{a}$ & 59,3 & 70,0 & 8,1 & 23,7 \\
Paclobutrazol 2 g/planta & 444,3 & 143,7 & $1097,5 \mathrm{~b}$ & 41,2 & 66,7 & 11,5 & 27,2 & 28,9 \\
Paclobutrazol 4g/planta & 471,3 & 150,8 & $1279,8 \mathrm{a}$ & 56,2 & 73,5 & 8,5 & 24,7 & 26,1 \\
Paclobutrazol 1000 mg/1 & 487,2 & 146,8 & $1185,7 \mathrm{ab}$ & 51,5 & 70,7 & 10,5 & 26,3 & 27,8 \\
foliar & & & & & & & & \\
Paclobutrazol 2000 mg/1 & 405,0 & 156,5 & $1157,5 \mathrm{ab}$ & 38,8 & 68,7 & 12,7 & 30,2 & 32,0 \\
foliar & & & & & & & & \\
Ane lamento simples & 405,0 & 136,2 & $1056,7 \mathrm{~b}$ & 37,2 & 60,0 & 11,0 & 23,4 & 30,0 \\
Anelamento duplo & 451,3 & 134,7 & $1067,0 \mathrm{~b}$ & 41,7 & 65,0 & 12,5 & 27,3 & 29,0 \\
\multicolumn{1}{c}{$\mathrm{F}$} & $\mathrm{NS}$ & $\mathrm{NS}$ & $5 \%$ & $\mathrm{NS}$ & $\mathrm{NS}$ & $\mathrm{NS}$ & $\mathrm{NS}$ & $\mathrm{NS}$ \\
\hline
\end{tabular}

As letras seguidas da mesma letra não diferem entre si, pelo teste de Duncan, ao nível de $5 \%$ de probabilidade. NS $=$ não-significativo

Rev. Bras. Frutic., Jaboticabal - SP, v. 30, n. 4, p. 857-861, Dezembro 2008 


\section{CONCLUSÕES}

1 - O anelamento diminuiu o crescimento de ramos e aumentou a produção de frutos.

2 - O paclobutrazol aplicado mais cedo via foliar não aumentou o número e o peso de frutos enquanto as aplicações via solo, aplicadas mais tarde, aumentaram a produção de frutos.

3 - $\mathrm{O}$ anelamento e o paclobutrazol não alteram a firmeza, o teor de nutrientes e os sólidos solúveis dos frutos.

4 - Os frutos maiores apresentaram maior teor de potássio.

\section{REFERÊNCIAS}

BRICKEL, C. A poda de Roseiras, arbustos de folhagem caduca e sempre-verde, sebes, arbustos de parede, arbustos de frutos, árvores de folha caduca. In: ENCICLOPÉDIA DE PRÁTICAS AGRÍCOLAS. Sociedade Real de Horticultura da Grã-Bretanha Publicações Europa América, 228 p. 1979.

CURRY, E. A.; WILLIAMS, M. W. Effect of paclobutrazol on fruit quality:apple,pear and cherry.Acta Horticulturae, Wageningen, n. 179, p743, 1986.

DECKERS, T.; SCHOOFS, H.; SMOLDERS, E. Natural or chemical regulation in pear. Acta Horticulturae, Wageningen, n.671, p. 503-516, 2005

ELFVING, D. C. Economic effects of excessive vegetative growth in decidous fruit trees. Hortscience, Alexandria, v. 23, p. 461-467, 1986.

FIORAVANÇO, J. C. A cultura da pereira no Brasil: situação econômica e entraves para o seu crescimento. Informações Econômicas, São Paulo, v.37, n. 39, 2007.

GLENN, D. M.; MILLER, S. S. Effects of Apogee on growth and Whole-Canopy Photosynthesis In Spur Delicious Apple trees. Hortscience, Alexandria, v. 40 n. 2 p. 397-400, 2005.

IUCHI, T.; IUCHI, V. L. Efeito do (Viviful 27,5\% prohexadione cálcio) sobre crescimento e frutificação da macieira cv Fuji Suprema. In: CONGRESSO BRASILEIRO DE FRUTICULTURA, 19., 2006, Cabo Frio. Anais... 355p.

LEOPOLD, A. C.; KRIDEMANN, P. E. Plant growth and development. New Delhi: Graw-Hill Publishing, 1975.545 p.

LUZ, R.; AZEVEDO, J.; CALOURO, F. Fertilização.Avaliação das necessidades nutritivas da pereira Rocha. In: ANP - Associação nacional de Produtos Pêra Rocha. Manual técnico de produção integrada de pêra Rocha. Cadaval, PRT, 2005. p. 29- 41.
MAAS, F. M. Shoot growth, fruit production and return bloon in "Conference" and "Doyenne" treated with Regalis (prohexadionecalcium). Acta Horticulturae, Wageningen, v. 671, p. 571-524, 2005.

MARAFON, A. C.; HERTER, F. G.; IUCHI, T.; HAWEROTH, F.J. Efeito do anelamento de tronco em pereira (Pyrus communis L.) cv. Abate Fetel na formação de gemas floríferas. In: CONGRESSO BRASILEIRO DE FRUTICULTURA, 20., ANNUAL MEETING OF THE INTERNATIONAL SOCIETY FOR TROPICAL HORTICULTURE, 54., 2008, Vitória, ES. Anais... CD-ROM

MEDJOUB, R.;VAL, J.; BLANCO, A. Inhibition of vegetative growth in red apple cultivars using prohexadione-calcium. Journal of Horticultural Science and Biotecnology, Kent, v.2, p.63-271, 2005. .

NACHTGALL; G. R.; BASSO, C.; FREIRE, C. J. H. Nutrição e adubação de pomares: maçã produção. Bento Gonçalves: Embrapa Uva e Vinho, 2004. p.63-78. (Frutas do Brasil)

NAVA, G.; BASSO,C.; NUENBERG, N.; MELO,G.W.; NACHTGALL., G.R; SUZUKI, A. Fertilidade do solo e nutrição na produção integrada de maçã. Bento Gonçalves: EMBRAPA, 2002. 13 p. (Circular Técnica, 33)

PRIVE, J. P.; FAVA, E.; EMBREE, C.; NICHOLS, D.; CLINE, J.; BYL, M.. Preliminary results on Efficacy of apple trees treated with the growth retardant prohexadione calcium (Apogee Regalis) in Eastern Canada. Acta Horticulturae, Wageningem, v. 636, p. 137-144, 2004.

SUZUKI, A.; ARGENTA, L. C. Teores minerais na polpa das cvs. Gala, Golden Delicious e Fuji. Revista Brasileira de Fruticultura, Jaboticabal, v. 16 p. 92-104, 1994.

WARNIER, O. Preliminary observations of effect of Regalis on apple and pear trees. Fruit Belge, Namur, n. 71, v. 502, p. 37-47, 2003.

WILLIAMS, M. W.; EDGERTON, L. J. Vegetative growth control on apple and pear trees with ICIPP 333. Acta Horticulturae, Wageningen, v. 137, p. 111-117, 1983.

YAMANISHI, O. K.; NAKAJIMA, Y.; HASEGAWA, K. Effect trunk strangulations in late Season on return,fruit quality and yield of pummelo tres grown in a plastic House. Japan Journal of the Japanese Society for Horticultural Science, Sakyo-Ku, v. 64, n.1, p31-40, 1995. 